AperTO - Archivio Istituzionale Open Access dell'Università di Torino

\title{
Ontology Development for Competence Assessment in Virtual Communities of Practice
}

\section{This is the author's manuscript}

Original Citation:

Availability:

This version is available http://hdl.handle.net/2318/1670012

since 2018-06-26T13:09:46Z

Publisher:

Springer

Published version:

DOI:10.1007/978-3-319-93846-2

Terms of use:

Open Access

Anyone can freely access the full text of works made available as "Open Access". Works made available under a Creative Commons license can be used according to the terms and conditions of said license. Use of all other works requires consent of the right holder (author or publisher) if not exempted from copyright protection by the applicable law. 


\title{
Ontology Development For Competence Assessment In Virtual Communities Of Practice
}

\author{
Alice Barana ${ }^{10000-0001-9947-5580]}$, Luigi Di Caro ${ }^{20000-0002-7570-637 X]}$, Michele Fiora- \\ vera $^{1 \text { [0000-0002-5150-6832] }}$, Marina Marchisio ${ }^{10000-0003-1007-5404]}$, Sergio Rabellino ${ }^{2[0000-0002-}$ \\ 1757-2000] \\ ${ }^{1}$ Department of Mathematics, University of Turin, Turin (TO), Italy \\ ${ }^{2}$ Department of Computer Science, University of Turin, Turin, (TO), Italy \\ alice.barana@unito.it \\ michele.fioravera@unito.it \\ marina.marchisiodunito.it \\ luigi.dicaroeunito.it \\ sergio.rabellinodunito.it
}

\begin{abstract}
This paper presents an ontological model for defining competency paths in STEM education, designed for the implementation of an adaptive system integrated in virtual communities. The model is applied for clustering materials for automatic assessment and the results are discussed.
\end{abstract}

Keywords: Automatic assessment, Competence, Ontology, Virtual community.

\section{Introduction}

The present work is part of a research whose aim is to enhance competency-based education. To foster learners' formative assessment and to support instructors in extending teaching strategies, a new system is proposed. Development and experimentation are conducted in Technology Enhanced Learning Environments (TELEs), ideal tool for managing large amount of data. Semantic-capturing methods are considered for automatically structuring resources by intended in-and-outcomes.

A methodology for preparing materials for automatic assessment is discussed considering results from ontology-based clustering of resources shared within the virtual community of a national-wide project for Secondary School.

\section{$2 \quad$ Methodology}

To enable automatic organization of any kind of resource, the system provides for natural language descriptions about intended in-and-outcomes. Models are applied for clustering a collection of resources produced and shared by instructors. The comparison between the original grouping and generated clusters are exploited for gathering insights about the applicability of the models.

Cite as: Barana A., Di Caro L., Fioravera M., Marchisio M., Rabellino S. (2018) Ontology Development for Competence Assessment in Virtual Communities of Practice. In: Penstein Rosé C. et al. (eds) Artificial Intelligence in Education. AIED 2018, pag. 94-98. Lecture Notes in Computer Science, vol 10948. Springer, Cham

https://doi.org/10.1007/978-3-319-93846-2 


\subsection{Models}

The new model of Measurable Learning Object is designed and proposed as essential authoring guide to explicate learning intentions and success criteria the materials are designed for. This model is linked to an ontological one, designed for extracting information about competences from free-texts. The Virtual Learning Community model specifies the context where the system can be implemented as integrated resource.

\section{Virtual Learning Community.}

The feasibility of the system relies on the existence of a common framework of competences expected to be achieved by learners at the end of the learning process, which instructors agree upon. Such framework can be efficiently explained and maintained in a Virtual Learning Community (VLC) $[1,2]$. It is a "community of communities": the community of instructors who collaboratively learn new methodologies supported by tutors in the use of advanced tools; the community of the online courses held by a teacher for his students; the global community of students.

The system is proposed to be experimented in national [3] and transnational [4, 5] scale, as integration of the Learning Management System hosting the VLC.

\section{Measurable Learning Object.}

This study focuses on the 'atomic' components of the products of Computer AidedAssessment [6], referred to as Measurable Learning Materials (MLO): digital resources containing one (only one) response area dedicated to automated assessment, associated to the "PRO" triple of descriptors:

- P-Performance ("instructional", "behavioural" or "learning" objective) is a specific statement about the observable behaviour required to the learner.

- $\mathrm{R}$ - Requisites ("pre-requisites") states the necessary and sufficient objectives that the learner is able to fulfil to successfully perform the MLO.

- O-Objectives (or "goals") specifies what the learner is required to be able to do as result of the educational activity the MLO was created for.

\section{Ontology.}

To extract knowledge from the descriptors' textual description, this research involves the use of an ontological version of Anderson \& Krathwohl taxonomy integrated with the domain-specific OntoMathPRO ontology (translated in Italian). OntoMathPRO is a bilingual (Russian/English) ontology of mathematical knowledge, shared with the Semantic Web community [7]. Anderson \& Krathwohl's classification organizes types of knowledge and thinking processes into categories [8].

Considering the ontological model, a MLO can be linked to a set of concepts' couples referring to a matrix: the first dimension of the matrix represents the types of knowledge while the second dimension represents the cognitive processes involved. The connection between a MLO and a matrix's element is established by identifying cognitive processes and knowledge type from its content or metadata. 


\subsection{Experimentation}

The models are applied for clustering a collection of MLOs by different features: the MLO's surface text and two versions of the MLO's Performance (P), Requisites (R) and Objectives $(\mathrm{O})$ authored separately by two experts. On each MLO's feature - which consists in an input string representing a descriptor or the surface text - the clustering process is performed by following 4 main phases.

- Tokenization, stop words removal, stemming, bag-of-words representation. Maple, the ACE on which Maple TA is based, is used for parsing surface text's formulae. To enhance the influence of semantically relevant concepts, this phase is repeated with tokens' filtering using the ontology:

- Words that appear in less than 2 input strings are filtered out.

- Words that appear in more than the half of the input strings are filtered out.

- Words recognized as ontological concepts are kept regardless the previous rules.

- After the previous rules, only the first n most frequent words are kept, with values of $n$ between the average lengths of vectors.

- The 'transformation model', initialized from the corpus of bag-of-words vectors, is used to convert any vector to the tf-idf representation.

- Mini Batch k-Means clustering algorithm [9] is executed on each feature's similarity matrices, constructed by calculating cosine similarity for each pair of vectors: each MLO is labelled with one out of k clusters, where $\mathrm{k}$ is equal to the number of "natural” MLOs' groupings by 'Disciplinary area' and by 'Response area type'.

V_measure homogeneity metric enables to estimate correlations between different clusterings [10]. Mean and standard deviation of the v_measure values from 10 process's repetitions are calculated. To evaluate whether clusterings correlation depends on the number of clusters generated, this is performed for different values of k. Clustering analysis is conducted by using tools from Gensim [11], nltk [12] and scipy [13].

\section{Results}

196 MLOs, produced with the Automatic Assessment System Maple TA [14] of the PP\&S VLC [15], were selected from problems shared by secondary school teachers.

Fig. 1 shows the mean of the v_measure values obtained comparing each of the 6 clusterings generated from the MLOs' descriptors of each given author $(1 \mathrm{P}, 1 \mathrm{R}, 1 \mathrm{O}$, $2 \mathrm{P}, 2 \mathrm{R}, 2 \mathrm{O}$ ) to respectively the labelling by disciplinary area (D) and by response area type (A), in case of $\mathrm{k}=8$ clusters to be generated, both without and with filtering considering values of $n$ between 7 and 15 in steps of 2 . The standard deviation values are about two orders of magnitude smaller than the means. Correlation values slightly increase/decrease with the increase/decrease of $\mathrm{k}$ from the number of D (and A) groupings, confirming the hypothesis of 8 clusters to be expected. Results highlight that clusterings generated by the descriptors highly reflect both D and A groupings, since the v_measure mean value is higher than 0.5 . These results align with the expectation that 
a MLO can be composed in potentially infinite modalities: the descriptors express concepts of mathematical problems referring to the mathematical model covered by the problematic situation. Filtering influences the descriptors accordingly with their respective average lengths of the generated vectors: it might enable to generate clusters which express concepts slightly different from those implicit in D or A.

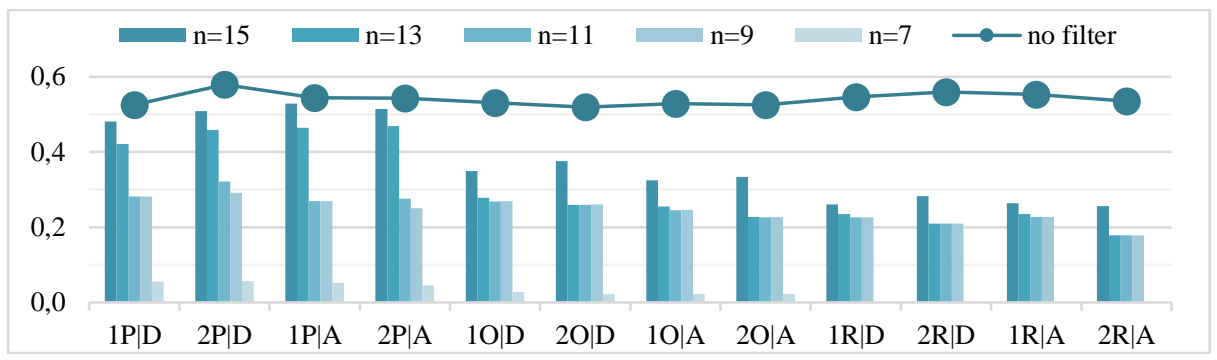

Fig. 1. Mean values of the $v_{-}$measure comparing $1 \mathrm{P}, 1 \mathrm{R}, 1 \mathrm{O}, 2 \mathrm{P}, 2 \mathrm{R}, 2 \mathrm{O}$ to $\mathrm{D}$ and $\mathrm{A}$, in case of $k=8$ clusters to be generated and values of $n$ between 7 and 15 in steps of 2 .

Correlation is less strong in comparison with the clustering generated by the surface texts: on average, the $\mathrm{v} \_$measure mean is less than 0.3 . To guarantee the quality of the clusterings obtained, $1 \mathrm{P}, 1 \mathrm{R}, 1 \mathrm{O}, 2 \mathrm{P}, 2 \mathrm{R}$ and $2 \mathrm{O}$ are compared to randomly generated clusterings: the v_measure mean values are significantly less than 0.1 .

Some correlation between descriptors is expected. Fig. 2 shows the mean of the v_measure values between different combinations of 1P, 1R, 1O, 2P, 2R and 2O. Results suggest significant correlation among Performance and Objectives of the same author, while Requisites appears to be highly independent.

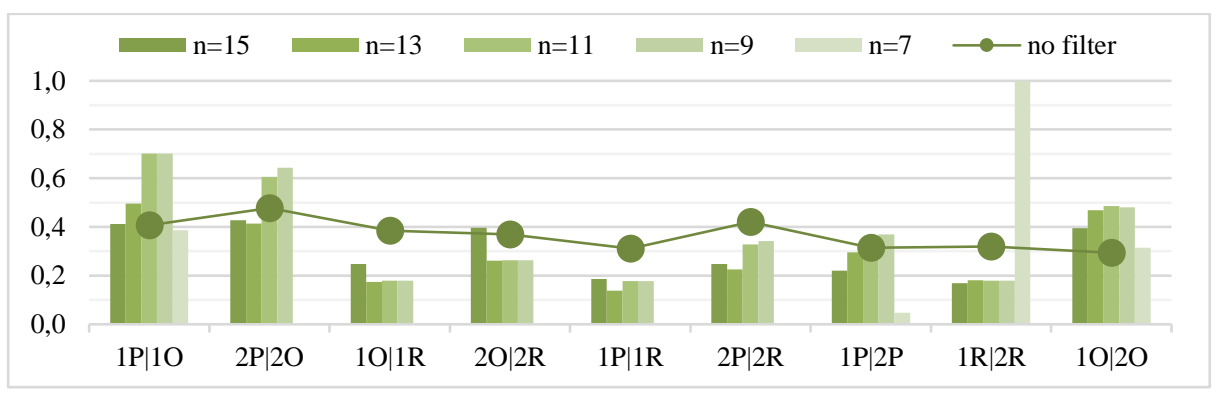

Fig. 2. Mean values of the $v \_$measure, for combinations of clustering from authors' descriptors, in case of $k=8$ clusters to be generated and values of $n$ between 7 and 15 in steps of 2 .

Only Objectives evidence stable inter-annotation agreement between the authors. Adopting ontologies as semantic-proxies would enable to capture semantically related concepts expressed with distinct words which generate differences between authors. Semantic measures based on ontologies will be the subject of further research towards the implementation of a system for adaptively providing learning resources. Ontology development will grow by activating projects at national and European scale. 


\section{References}

1. F. Ellerani and M. Parricchi, “Ambienti per lo sviluppo professionale degli insegnanti”, Web 2.0, gruppo, comunità di apprendimento, 2010.

2. C. Demartini, M. Marchisio and C. Pardini, "PP\&S100 - una comunità di comunità di collaborative learning attraverso le nuove tecnologie", Atti Didamatica Pisa 2013, pp. 989-998, 2013.

3. A. Barana, M. Fioravera and M. Marchisio, Developing problem solving competences through the resolution of contextualized problems with an Advanced Computing Environment, Proocedigs of the $3^{\text {rd }}$ International Conference on Higher Education Advances (HEAd'17), Valencia, pp. 1015-1023, 2017.

4. A. Brancaccio, M. Esposito, M. Marchisio and C. Pardini. "L'efficacia dell'apprendimento in rete degli immigrati digitali. L'esperienza SMART per le discipline scientifiche”, Mondo Digitale, 15, ISSN: 1720-898X, pp. 803-821, 2016.

5. A. Brancaccio, M. Marchisio, C. Meneghini and C. Pardini. "Matematica e scienze più smart per l'insegnamento e l'apprendimento", Mondo Digitale, XIV, 58, pp. 1-8, 2015.

6. A. Barana and M. Marchisio, "Ten good reasons to adopt an automated formative assessment model for learning and teaching Mathematics and scientific disciplines", Procedia: Social \& Behavioral Sciences, 228, doi:10.2016/j.sbspro.2016.07.093, pp. 608-613, 2016.

7. O. Nevzorova, N. Zhiltsov, Nikita, A. Kirillovich and E. Lipachev, "OntoMathPRO ontology: A linked data hub for mathematics. Communications in Computer and Information Science", 468, 2014.

8. L.W. Anderson, D.R. Krathwohl et al., "A taxonomy for learning, teaching, and assessing. A revision of Bloom's taxonomy of educational objectives", New York, Addison Wesley Longman, 2001.

9. F. Pedregosa, G. Varoquaux, A. Gramfort, V. Michel, B. Thirion, O. Grisel, M. Blondel, P. Prettenhofer, R. Weiss, V. Dubourg, J. Vanderplas, A. Passos, D. Cournapeau, M. Brucher, M. Perrot and E. Duchesnay, "Scikit-learn: Machine Learning in Python". JMLR 12. 28252830, 2011.

10. A. Rosenberg and J. Hirschberg, "V-Measure: A conditional entropy-based external cluster evaluation measure", Proceedings of the 2007 Joint Conference on Empirical Methods in Natural Language Processing and Computational Natural Language Learn-ing(EMNLPCoNLL), pp. 410-420, 2007.

11. R. Řehůřek and P. Sojka, "Software Framework for Topic Modelling with Large Corpora", Proceedings of LREC 2010 workshop New Challenges for NLP Frameworks, Valletta, Malta: University of Malta, pp. 46-50, 2010.

12. S. Bird, L. Edward and K. Ewan, "Natural Language Processing with Python", O'Reilly Media Inc, 2009.

13. Jones E., Oliphant E., Peterson P., et al. SciPy: Open Source Scientific Tools for Python, 2001-, http://www.scipy.org/ [Online; accessed 2018-01-29].

14. A. Barana, M. Marchisio and S. Rabellino, "Automated Assessment in Mathematics". IEEE $39^{\text {th }}$ Annual Computer Software and Applications Conference (COMPSAC), doi: 10.1109/COMPSAC.2015.105, pp.670-671, 2015.

15. A. Brancaccio, C. Demartini, M. Marchisio, C. Palumbo, C. Pardini, A. Patrucco, R. Zich, "Problem Posing and Solving: Strategic Italian Key Action to Enhance Teaching and Learning of Mathematics and Informatics in High School”, IEEE $39^{\text {th }}$ Annual Computer Software and Applications Conference (COMPSAC), doi: 10.1109/COMPSAC.2015.126, pp. 845$850,2015$. 